\title{
Growth Mechanism and Characteristics of Semiconductor Nanowires for Photonic Devices
}

\author{
N. B Singh ${ }^{1}$, S. R Coriell ${ }^{1}$, Matthew King ${ }^{2}$, Brian Wagner ${ }^{2}$, David Kahler ${ }^{2}$, David \\ Knuteson ${ }^{2}$, Andre Berghman ${ }^{2}$ and Sean McLaughlin ${ }^{2}$ \\ ${ }^{1}$ University of Maryland Baltimore County, 1000 Hilltop Circle, Baltimore, MD 21250 \\ ${ }^{2}$ Northrop Grumman Corporation ES, 1212 Winterson Road, Linthicum, MD 21250
}

Received: March 31, 2014; Accepted: April 27, 2014; Published: April 29, 2014

*Corresponding author: N. B Singh, University of Maryland Baltimore County, 1000 Hilltop Circle, Baltimore, MD 21250

\begin{abstract}
An overview on the growth mechanism of nanowire, fabrication and performance of selenide nanowires devices is presented. Binary, ternary and quaternary selenides have relatively low optical absorption coefficient and have wide transparency range. This combination of properties of selenides makes them very important materials for detector applications. Lead selenide ( $\mathrm{PbSe}$ ) nano particles and nanowires were grown by the physical vapor transport (PVT) method and zinc selenide ( $\mathrm{ZnSe}$ ) nanowire was grown by chemical vapor transport (CVT) methods. Observations on the growth of PbSe indicate that oriented attachment of nanocrystal building blocks produces nanowires. On silicon (111) substrates binding was observed to be on (001) and (111) faces. Zinc selenide nanowires were fabricated into nano Avalanche Photodiodes (Nano-APDs) structure. Experimental results on the fabricated devices of zinc selenide show significant enhancement in photoconductivity. Growth of nanowire and fabrication of devices has progressed significantly and has shown significant improvements in the performance, but fabrication still remains a big challenge.
\end{abstract}

Keywords: PbSe; ZnSe; Nanowire growth; Vapor deposition; Photodetector; Fabrication

\section{Introduction}

The emergence of nanotechnology has provided a great deal of promise for the area of low light collection due to small size and hence decreased dark current. Since applications of nano-APDs in the short wavelength infrared (SWIR) region has gained a lot of momentum, significant progress has been made [1-7] by several research groups. Nano-APDs have significant advantages over traditional night vision equipment which generally amplifies radiation over the visible and near infrared range out to a wavelength of $1 \mu \mathrm{m}$. The near-infrared (NEIR) to mid-wave infrared (MWIR) $\mu \mathrm{m}$ region is an excellent choice since it extends beyond the cutoff of silicon detectors. In GaAs, PbS, and PbSe based detectors cover short wave infrared (SWIR), NEIR and MWIR region. But there is a big gap between the MWIR and long wave infrared (LWIR) region. High gain and fast response time of APDs is required for variety of applications. NWs have shown promising optical properties, including high transparency and a high order of magnitude better photocurrent. Avalanche photodiodes (APDs) are very capable detectors in finding and photon counting applications. These arecapable of low-light detectionand have great potential for the variety of applications. Growth of nano particles and preliminary results on the formation of nanowires of lead selenide is reported. Results are presented for the zinc selenide nanowire based devices, performance and more specifically the ability to transition from single NW devices to device arrays.

\section{Experimental Method}

\section{Materials purification}

As supplied $\mathrm{PbSe}$ and $\mathrm{ZnSe}$ materials had listed purity of 99.995\%. These materials were purified by physical vapor transport method in a cleaned quartz tube in vacuum. We have used both horizontal and vertical geometry. We always observed dark residue left behind after evaporation. In order to ensure to avoid the moisture we heated samples at $120^{\circ} \mathrm{C}$ before starting the purification. The evaporation temperature was above $300^{\circ} \mathrm{C}$ in vacuum.

\section{Nanowire growth}

We have used both physical vapor transport (PVT) and chemical vapor transport (CVT) growth methods for the growth of nanoparticles and nanowires. Details of these methods are described by authors elsewhere [7-8]. A limited number of growth experiments were performed using vapor-liquid-solid method similar to method described by Cho et al [9] to compare with PVT results. Before starting the growth, the vacuum system was sufficiently pumped and purged prior to growth to eliminate oxygen contamination. For the growth of lead selenide nanoparticles physical vapor transport growth was used at a source temperature range of $300^{\circ} \mathrm{C}$ to $400^{\circ} \mathrm{C}$ and for nanowires temperature was maintained below $300^{\circ} \mathrm{C}$. Silicon wafers (111) with thermal oxide with gold catalyst were used as substrates for $\mathrm{PbSe}$ system. For the large area silicon wafers (>3inch diameter) we used commercial DENTON evaporator. However for the PVT and vapor-liquid-solid and smaller diameter substrates $(<1$ inch silicon) a three zonehorizontal transparent glass furnace was used. 
The growth chamber is described in Matthew $\mathrm{R}$ et al. [7] and is shown in Figure 1. For the case of ZnSe Source temperatures ranged from $750^{\circ} \mathrm{C}-1000^{\circ} \mathrm{C}$, while the substrate temperature range was $675^{\circ} \mathrm{C}-925^{\circ} \mathrm{C}$. Argon gas was used to purge the growth chamber. For the understanding the growth morphology and dimension of the nanowirewe used a partial pressure range of Argon between 250mTorr - 760Torr. Sapphire substrates were used for ZnSe growth. Typical cleaning procedures were used to prepare the substrates for growth. Gold catalysts were then deposited on the substrates and the diameter for the catalyst was in the range of $10-50 \mathrm{~nm}$.

\section{Characterization and fabrication}

X-ray characterization was performed for Omega-2 The ta scan to determine the NW peak positions. We used a Bede D1 x-ray diffractometer equipped with a micro source and a triple-bounce Ge crystal beam conditioner. The quality and the orientation of the material were determined by X-ray rocking curve and composition was determined by XPS. It was very clear that PVT process produces much better materials than vapor-solution-solid growth methods since impurities of the solution affect the growth. The morphology of nano structure was studied using a Hitachi FE-SEM equipped with a PGT EDS. Optical morphology was studied using a microscope coupled to an Ocean Optics USB4000-XR for determining the transmission properties of single wire. Photoluminescence was studied using $266 \mathrm{~nm}$ light source. Performance of the fabricated nanowire devices were evaluatedby current-voltage data in the range of -20 to $20 \mathrm{~V}$. A $150 \mathrm{~W}$ broadband halogen light was used as a source on the device through the microscope objective on the probe station. Current-voltage curve was used to determine the ratio of photocurrent to dark current for each NW device.

\section{Results and Discussion}

It is well established that nucleation produces the desired shapes, and size can be controlled by controlling the growth rate and temperature, in the case of pure and doped lead selenide transition to nanocubes has added another complexity for achieving the desired sizes of nanowire for fabrication. In order to understand this in details we describe the growth mechanism of nanowires.

\section{Growth mechanism of nanowires}

It is extremely difficult to compare growth results of nanowires with any theory since impingement of atoms on substrates, nucleating agent, tip shape, anisotropy interaction of one nanowire and several other parameters affect the growth rate and morphology. The classical model of whisker growth was based on impingement of atoms on the whisker sides, and diffusion of these adatoms to the whisker tip where they are incorporated in the growing whisker. The vapor-liquid-solid (VLS) method used a liquid drop at the whisker tip which was an effective method of capturing atoms from the vapor. Parker et al [10] and Givargizov et al [11] have measured $\mathrm{L}(\mathrm{t})$ as a function of time $t$ for a fixed whisker radius $r$. For nanowires it is clearly difficult to measure $\mathrm{L}(\mathrm{t})$ during a growth experiment.

A typical measurement consists of carrying out an experiment for a fixed time and after stopping the growth process, measuring the nanowire length $L$ as a function of the nanowire radius $r$. Johansson et al.[12] used this method for data on GaP nanowires and Schmidt et al [13] used the method for Si nanowires studies. Johansson et al. [12] also present a simple mass transport model of nanowire growth. The model includes impingement of atoms on the substrate, on the nanowire sides, and on the liquid drop. The atoms on the substrate diffuse [14] to the whisker base and

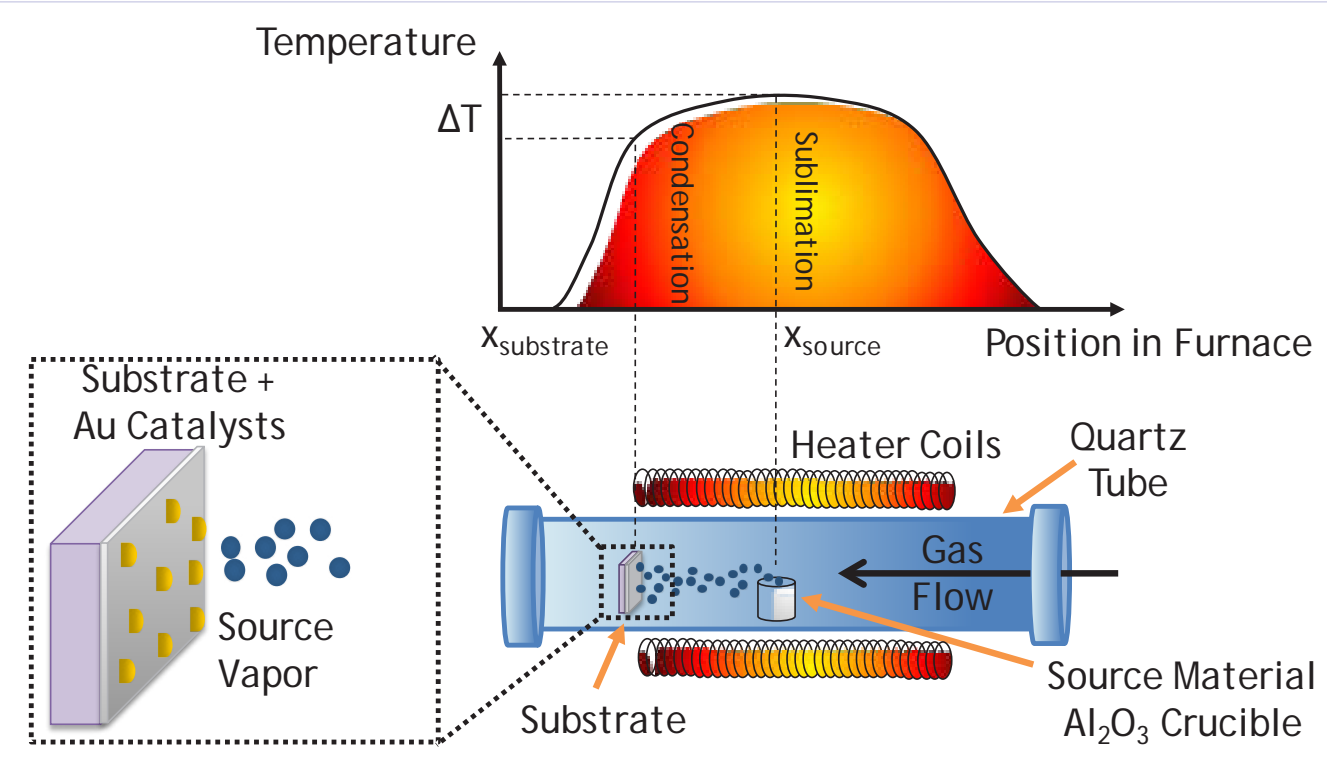

Figure 1: Schematic of Nano wire growth furnace is described by King et al [7] in details for controlling the thermal profile, vapor pressure and to achieve the size of the nanowires. 
then along the whisker sides to the liquid drop where they are incorporated into the nanowire. Johansson et al. [14] also present a simple mass transport model of nanowire growth. The model includes impingement of atoms on the substrate, on the nanowire sides, and on the liquid drop. The atoms on the substrate diffuse to the whisker base and then along the whisker sides to the liquid drop where they are incorporated into the nanowire. The model results in an expression for the nanowire growth velocity $\mathrm{dL} / \mathrm{dt}$ as a function of the nanowire length $\mathrm{L}$. An integration of this expression yields $L$ for a given processing time. Johansson et al [14] use this model to fit nanowire length as a function of nanowire radius data. The model involves three impingement rates (substrate, nanowire sides, and liquid droplet) and two surface diffusion lengths (substrate and nanowires sides). Assuming that all the impingement rates are the same and that the surfaces diffusion lengths are the same greatly reduces the number of parameters. The model is such that one can always pick an impingement rate to obtain the nanowire length. The experimental data and theory indicate that the dependence of nanowire length on nanowire radius is complicated and depends on the material and growth conditions. There are also models similar to that of Dubrovskii et al [15] that take into account diffusion through the liquid drop (or on the surface of the liquid drop) and also account for the difficulty of incorporation into the nanowire (interface kinetics). These more sophisticated models involve a number of thermophysical properties that are usually not well known for specific materials. Dubrovskii et al. [15] also obtain a differential equation for the change in nanowire radius with time (tapering of the nanowire) based on changes in the size of the liquid drop due to changes in the solute composition in the drop. There is also experimental evidence supported by studies of Johansson et al. [14] that for some materials nanowire growth occurs only when the drop is solid not liquid. The mass transport model of Johansson et al [12] yields an equation for the growth velocity of the nanowire

$$
\frac{1}{2 \Omega} \frac{d L}{d t}=\frac{R_{w} \lambda_{w}}{r_{w}} \tanh \left(L / \lambda_{w}\right)-\frac{J_{s w}}{r_{w} \cosh \left(L / \lambda_{w}\right)}+R_{\text {top }},
$$

Where $\mathrm{L}$ is the length of the nanowire and $\mathrm{t}$ is time. $J_{s w}$ is the adatom flux from the substrate to the nanowire and is given by

$$
J_{s w}=-R_{s} \lambda_{s} \frac{K_{1}\left(r_{w} / \lambda_{s}\right)}{K_{0}\left(r_{w} / \lambda_{s}\right)} .
$$

Here $\Omega$ is the atomic or molecular volume, $R_{w}$ is the deposition rate on the wire sides, $\lambda_{w}$ is the diffusion length on the wire sides, $r_{w}$ is the radius of the wire, $\lambda_{s}$ is the diffusion length on the substrate, $K_{0}$ and $K_{1}$ are modified Bessel functions, $R_{s}$ is the deposition rate on the substrate, and $R_{\text {top }}$ is the deposition rate on the top of the wire (or on the liquid drop). Atoms on the substrate diffuse to the nanowire base and then along the sides of the nanowire to the top of the nanowire. The Bessel functions arise from the solution of the steady state diffusion equation on the substrate. The diffusion solution assumes that the separation between nanowires is large compared to the diffusion distance $\lambda_{s^{*}}$ The model neglects any resistance (interface kinetics) to incorporation of atoms into the nanowire crystal.

The time does not appear explicitly on the right hand side of
Eq. (1) so it has the form

$$
\frac{1}{2 \Omega} \frac{d L}{d t}=f(L)
$$

where $f(L)$ denotes the right hand side of Eq. (1). Hence, we have

$$
2 \Omega t=\int_{0}^{L} \frac{d L}{f(L)} .
$$

In general the integral requires numerical evaluation. If all of the deposition rates are equal to

$$
\begin{aligned}
& \mathrm{R}\left(R_{s}=R_{w}=R_{\text {top }}=R\right) \text {, then we have } \\
& 2 \Omega R t=\int_{0}^{L} \frac{d L}{g(L)},
\end{aligned}
$$

Where $g(L)$ is given by

$$
g(L)=\frac{\lambda_{w}}{r_{w}} \tanh \left(L / \lambda_{w}\right)+\frac{\lambda_{s} K_{1}\left(r_{w} / \lambda_{s}\right)}{r_{w} K_{0}\left(r_{w} / \lambda_{s}\right) \cosh \left(L / \lambda_{w}\right)}+1 .
$$

Eq. (5) specifies $2 \Omega R t$ for a given value of the nanowire length.

For the limiting cases in which there is deposition on only one of the surfaces, the integral in Eq. (4) can be done analytically. For deposition only on the top $R_{w}=R_{s}=0$, we have the simple result

$$
L=2 \Omega R_{\text {top }} t .
$$

The nanowire length $\mathrm{L}$ is linear in time. The factor 2 arises from the assumption that the liquid drop at the tip of the nanowire is a hemisphere which has twice the area of the nanowire tip.

For deposition only on the whisker sides $R_{s}=R_{\text {top }}=0$, we have

$$
\sinh \left(L / \lambda_{w}\right)=\sinh \left(L_{0} / \lambda_{w}\right) \exp \left(2 R_{w} \Omega t / r_{w}\right),
$$

Where $L_{0}$ is the nanowire length at $\mathrm{t}=0$. In this limit there must be an initial whisker length to cause growth. The above expression is the Dittmar-Neumann approximation to the classical whisker growth problem.

For deposition only on the substrate $R_{s}=R_{\text {top }}=0$, we have

$$
\sinh \left(L / \lambda_{w}\right)=-\frac{2 \Omega J_{s w} t}{r_{w}} .
$$

When the length of the nanowire has become sufficiently long that $L>>\lambda_{w}$ and $L>>\lambda_{s}$, Eq. (1) can be approximated by

$$
\frac{1}{2 \Omega} \frac{d L}{d t}=\frac{R_{w} \lambda_{w}}{r_{w}}+R_{\text {top }} .
$$

In this limit deposition on the substrate no longer contributes to the nanowire growth.

For no deposition on the substrate, it is possible to obtain a more general result. Integration of Eq. (4) for $R_{s}=0$ yields

$$
\frac{2 \Omega t}{\lambda_{w}}\left[\left(\frac{R_{w} \lambda_{w}}{r_{w}}\right)^{2}-R_{\text {top }}^{2}\right]=-\frac{R_{\text {top }} L}{\lambda_{w}}+\frac{R_{w} \lambda_{w}}{r_{w}} \ln \left[\frac{\sinh \left[\beta+\left(L / \lambda_{w}\right)\right]}{\sinh \beta}\right],
$$

Where the constant $\beta$ is defined by $\tanh \beta=R_{\text {top }} r_{w} /\left(R_{w} \lambda_{w}\right)$. The above form is appropriate when $R_{\text {top }} r_{w} /\left(R_{w} \lambda_{w}\right)<1$.

A few calculations have been carried out for the experiments of Johansson et al. [12] on GaP nanowires. The integral in Eq. (4) 
was evaluated numerically for $R_{s}=R_{w}=R_{\text {top }}=R$ and $\lambda_{w}=\lambda_{s}=300$ $\mathrm{nm}$. A value of $2 \Omega \mathrm{R} t=96 \mathrm{~nm}$ was chosen to provide a reasonable fit to the experimental data. The values of the deposition rate and growth time were not given in their article. The calculated nanowire lengths were 2222, 1338, $990 \mathrm{~nm}$ for wire radii of 20 , 40 , and, $60 \mathrm{~nm}$, respectively. The decrease of wire length with increasing wire radius is in reasonable agreement with Johansson et al. For a wire radius of $40 \mathrm{~nm}$, if $R_{\mathrm{s}}$ is set to zero, a nanowire length of $343 \mathrm{~nm}$ is obtained, while if $R_{w}=R_{\text {top }}=0$, a nanowire length of $840 \mathrm{~nm}$ is obtained. As shown in Figure 2, for a free standing PbSe nanowire of the diameter of $200 \mathrm{~nm}$ we observed a length of $180 \mathrm{~nm}$.

\section{Lead Selenide nanowires}

Significant experimental data on the morphology of semiconductor nanowires, nanocubes and nanoparticles have been reported [9, 16-17]. Lieber et al [17] have reported results of nanowire growth as a function of growth time. Their results have shown that nanowire length is directly proportional to growth time and have demonstrated that indium phosphate nanowires with narrow length distributions centered at 2, 4, 6, and $9 \mathrm{~nm}$. They indicated that new level of synthetic control and our approach should enable better-defined fundamental studies of nanowires and open up new opportunities for the assembly of functional nanodevices. Later Cho et al [9] demonstrated good uniformity of nanowire diameter along the entire length.

Results reported here show the nanodots and arrays of nanocubes on Silicon $(\mathrm{Si})$ wafers which were grown with and without catalyst. Silicon (111) wafer was used as the substrate for the growth. Several growth experiments were carried out and growth conditions were varied to study the nucleation and growth of dots. The morphologies of the nanodots were very much dependent on the growth conditions. We observed that

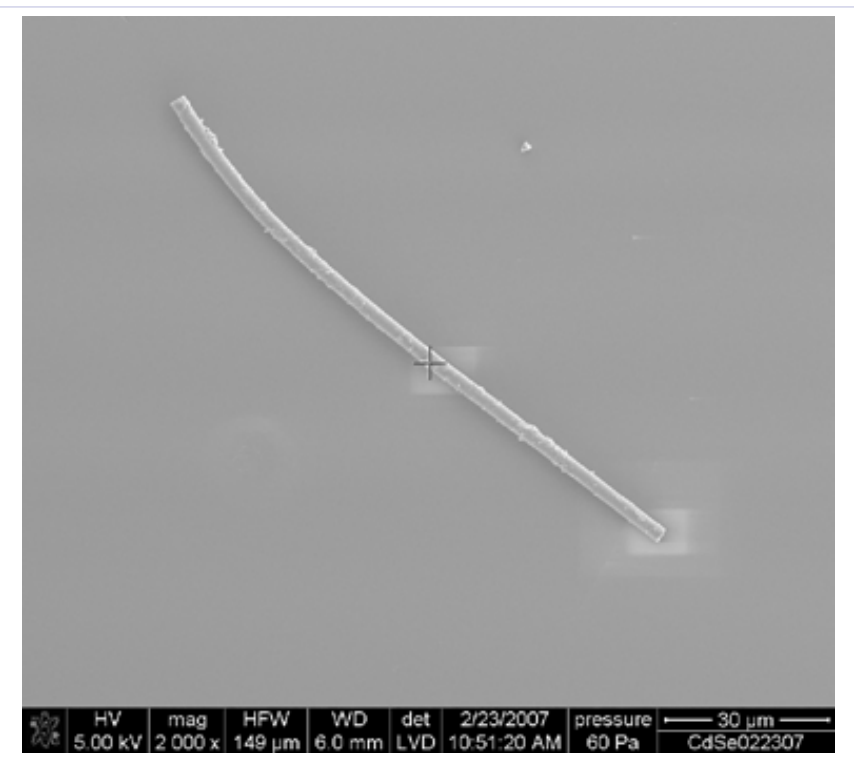

Figure 2: (a) A single PbS nanowire growing in uniform diameter (b) Growth of $\mathrm{PbS}$ nanowire for which diameter is affected by presence of neighboring nanowire. the morphology was nanocubes and nanodots on wafers with and without catalyst. Larger oriented PbSe dots grew at high substrate temperature. Effect of substrate modification was observed by putting the nickel silicide film on Si wafers. Growth experiments indicated that using silicides the growth morphology changed significantly. Wire diameters and length were measured using SEM images. The quality of the material was determined by X-ray rocking curve and composition was determined by XPS. Results are shown in Figure 3. Also, we observed the transition of morphology from nanoparticle into nanocubes shown in Figure 4.

Most of the nanowire growth studies have been performed using vapor-liquid-solid conditions and transferred on wafers using fluids. One can achieve large nanowires and results can be compared with theories described in section 3.1. In these experiments wafer surfaces (roughness, interface energy, impingement and impurities) do not influence the growth and kinetics. However for low cost practical applications direct growth on wafer is an ideal approach. Figure 4 shows the growth of impinged nanoparticles of PbSe nanocubes on Silicon wafer and morphology of nanocubes. These nanocubes are very much oriented. At some places these nanocubes are attached with each other and form very small nanowires. If conditioned are favorable, the growth of nanocubes continues to form the nanowires. We observed this phenomenon was more favored in lead salts in presence of other impurities. Previous studies including that of Cho et al [9] show that PbSe nanocrystals bind to each other on either $\{100\},\{110\}$, or $\{111\}$ faces depending on the molecules present in the reaction solution. The noncentrosymmetric arrangement of $\mathrm{Pb}$ - and Se-terminated [111] facets and possess dipole can also be the driving mechanism for one-dimensional oriented attachment of nanocrystals to form nanowires. Both surfactants and dipole can be easily altered in the solution method compared to that of PVT process. This is the reason why materials such as oleic acid enable significant changes in the shape and diameter of lead selenide nanowires. Effect of binary and multinary surfactants is more complex. More studies are required to achieve long and thin nanowires to demonstrate role of interface energy and mechanism of these materials. Also, although growth of nanocubes indicated [Figure 5] strong orientation in (001) and (111) orientation, further research is needed to clarify the strong tendency of nanowire growth in (110) direction for PbSe nanowires.

\section{ZnSe nanowires}

ZnSe nanowires were also grown and fabricated into photodetectors. We successfully synthesized nanowires into an aligned array and results are summarized in Figure 6 Matthew $\mathrm{R}$ et al. [7]. As discussed in the section of growth mechanism of nanowires the alignment is preferential to both the substrate surface and the NW array as a whole. The desired size of nanowire was achieved by controlling the experimental parameters. No post-processing or ex-situ alignment methods were used. As is typically the case with VLS growth mechanism, we observed that the catalyst diameter largely determined the subsequent NW diameter. NW length on the other hand depends largely on the 


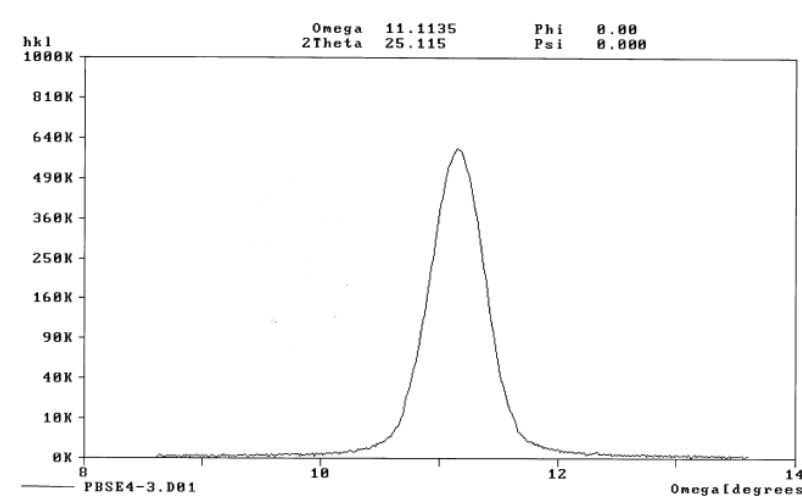

(a)

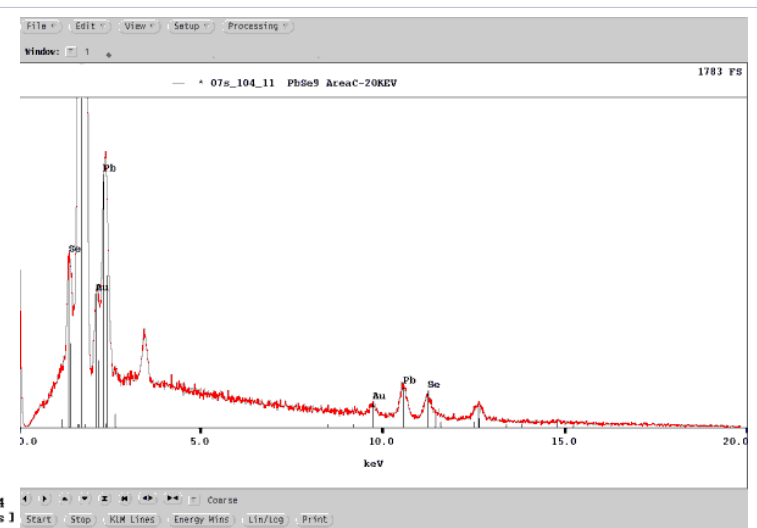

(b)

Figure 3: (a) 2 Theta-Omega curve showed the quality of the material and (b) compositional analysis did not show any impurity.
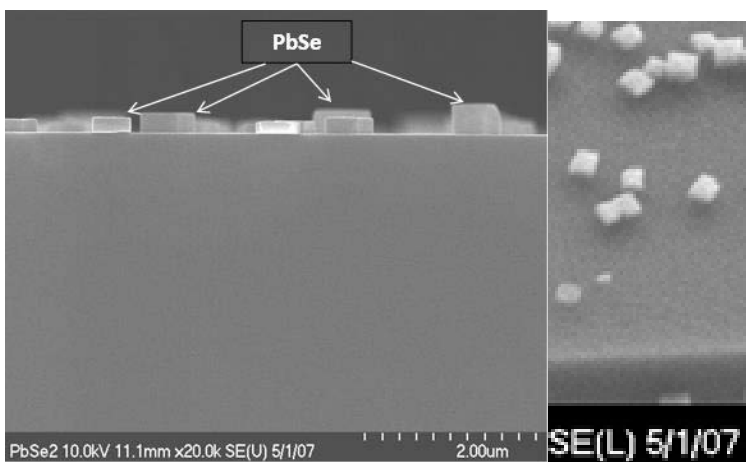

(a)

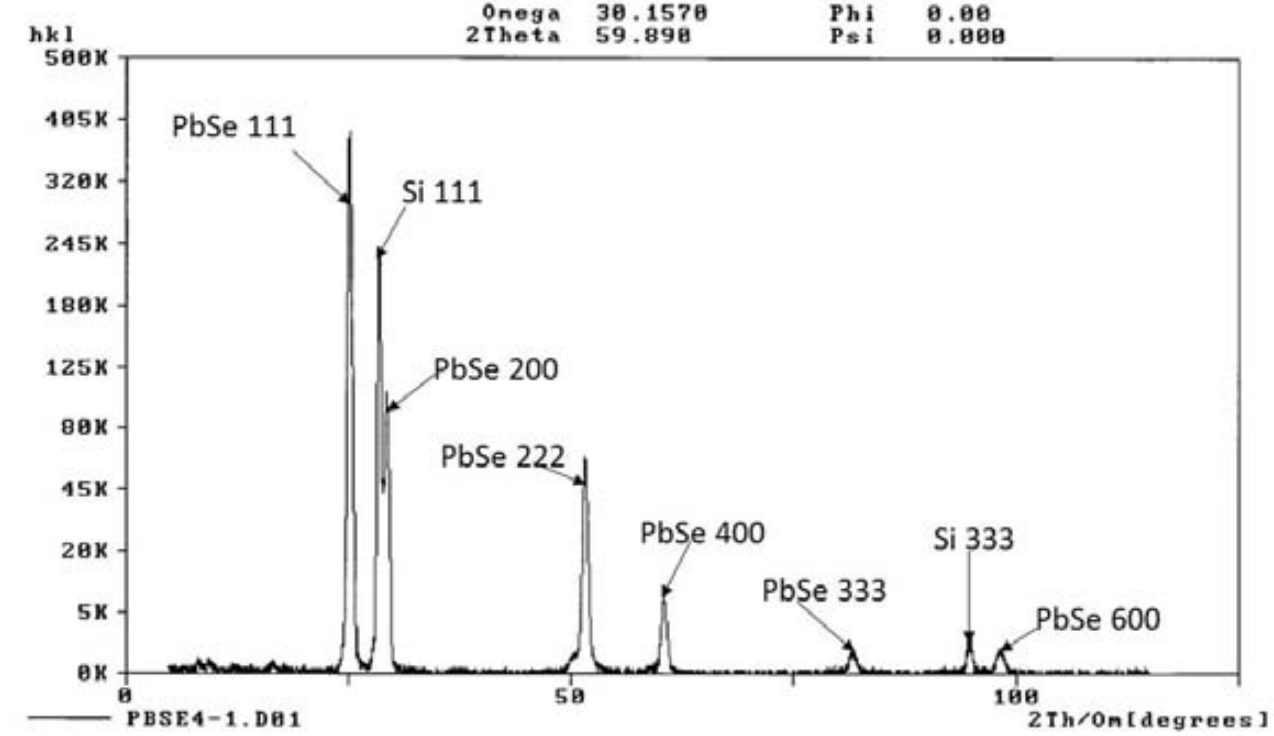

Figure 5: PbSe grown at low temperature showed both (001) and (110) orientation. However growth at high temperature favored (001) growth. 


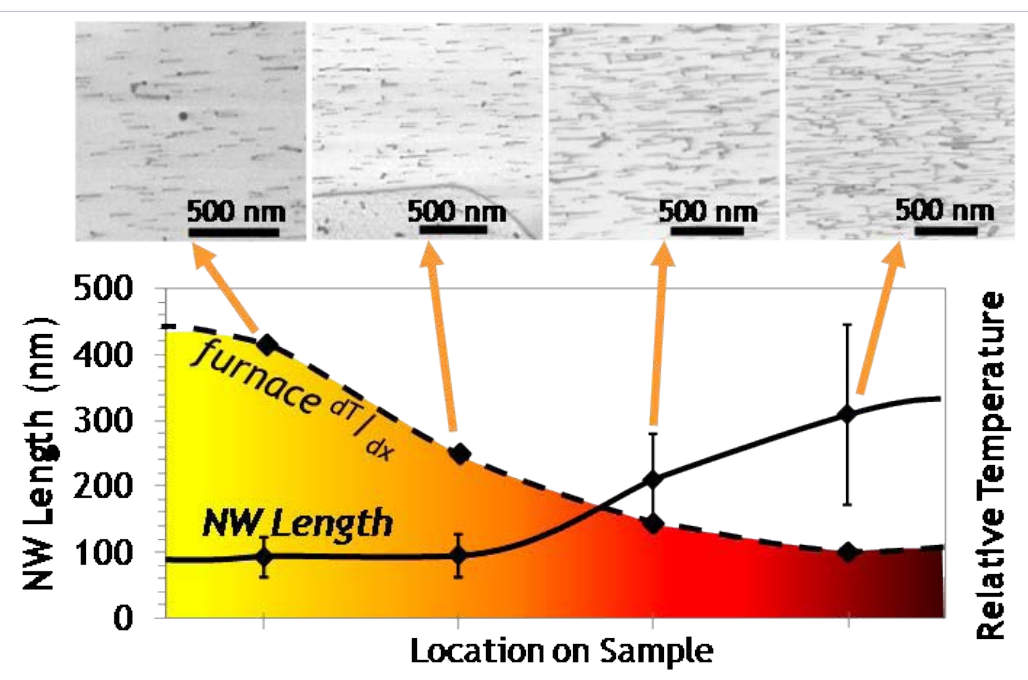

Figure 6: Schematic comparison of ZnSe NW length to the relative thermal gradient in the furnace. NW length is seen to increase with decreasing temperature, or higher $\Delta \mathrm{T}$. (7).

growth conditions. Details on the effect of growth conditions on the morphology and size are discussed in Matthew $\mathrm{R}$ et al. [7]. SEM studies indicated that NW lengths were consisted of $30-40 \mathrm{~nm}$ at a given sample location. The sample apexshows the highest average NW length due to coldest location on the sample. This indicated an inverse relationship exists between substrate temperature and resulting NW length. This can be also explained on the basis of the sticking coefficients. At higher temperatures, the sublimed vapor had sufficient energy to desorb from the substrate surface following condensation. At reduced temperatures the atoms may have enough thermal energy to diffuse on the surface but are limited in their ability to transition back to the vapor phase. ZnSe showed the highly preferential growth direction of (111), especially with respect to the crystallography of the underlying substrate. This was further confirmed by an offset in $\mathrm{ZnSe}$ (111) peak position resulting from epitaxial matching of the mis cut substrate.

ZnSe NW arrays were characterized by photoluminescence spectroscopy. PL spectra showeda strong near band edge (NBE) emission at $463 \mathrm{~nm}$. This is a typical of the high luminosity inherent in the ZnSe nanowires grown by the physical vapor transport method. This is due in large part to strong excitonic interaction between photogenerated charge carriers the $\mathrm{ZnSe}$ exciton binding energy $\sim 21 \mathrm{meV}$. A low intensity broad emission band centered around $\sim 650 \mathrm{~nm}$ was also observed. This was due to the deep level defects. This deep level defect is a good measure of the quality of nanowires. In this case since the quality of nanowire was very good, this deep level defect band was very low intensity. The data was also supported by XRD data.

\section{Device design and fabrication}

Using NWs, one can design and develop homogeneous composition and single-crystal structures for nano devices. The ability to control the fundamental electronic properties of NWs through doping has been central to much of our success in developing active electronic and optoelectronic nanodevices, where the composition and/or doping is modulated down to the atomic level along or perpendicular to the axes of NWs.

Almost all work [1-7] on Nano-APDs is focused because the dark current of the Geiger-mode APDs increases with size due to thermally generated carriers. Based on these results and the small nano-APD size one can conclude that capacitances and series resistances of nano-sized APDs are much lower than their micron-sized counterparts. As the capacitances (C) and series resistances (R) of nano-sized APDs are much lower than their micron-sized counterparts, the smaller $R C$ time constant reduces the recovery time for passive quenching and the gated length for gated passive quenching, which are important for minimizing DCR. Since the total RC constant (which includes control circuitry contributions) determines the band width in optimized APD designs, higher bandwidth is expected for nano-APDs. A variety of designs are proposed for nano-APDs. The main design involves crossed $\mathrm{p}$ - and n-type nanowires grown on a Si substrate, with the nano-APD defined by the nanoscale $p$ - $n$ junction where the two wires meet. By growing n-type nanowires directly on p-type substrates or epitaxially grown film, the fill factor within a pixel increases and sensitivity improves.These design variations optimize performance and assist with achieving single photon detection. This design shown in Figure 7 was used for evaluating the quality of nanowires. A large number of experimentshave beenperformed on the growth and ZnSe Nanowire based APDs. After the growth of ZnSe nanowires, electrode arrays were fabricated using standard semiconductor process. Currentvoltage data were used to determine the device characteristics and the sensitivity of the optical light. ZnSe nanowires showed strong photoactivity on exposure of the light source. The measurement of photo current for a 40-device array showed an average increase in current after annealing in the Ar atmosphere. The measured values for photocurrent ratios were more than 700 times higher. Devices showed (Figure 8) typical characteristics of Schottky contacts. 


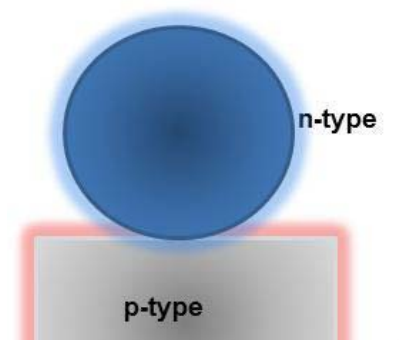

NW on Epi $(p-n)$
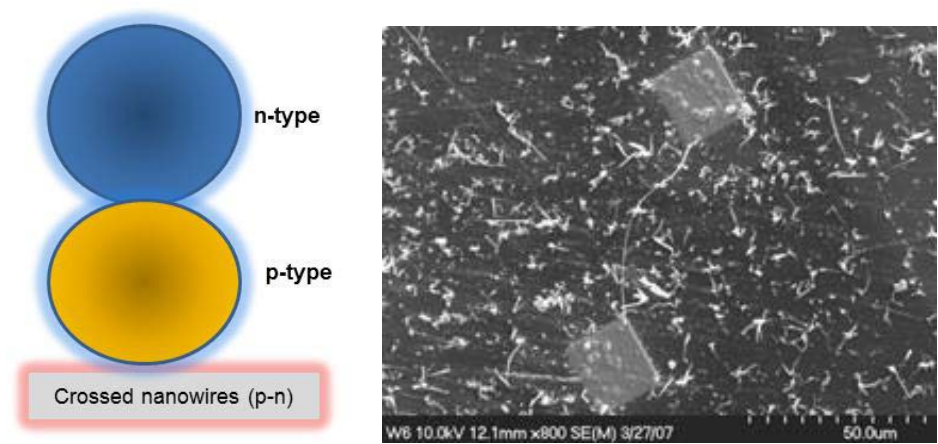

Figure 7: Schematics of two designs; n-type wire on p-type substrate and n-type nanowire on a p-type nanowire in cross wire design, and contacts for a n-type wire on Si-substrate.

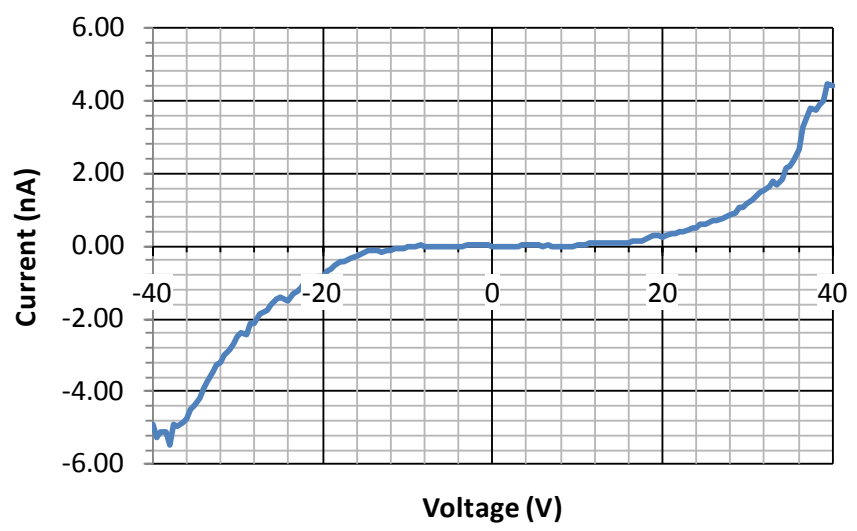

Figure 8: Fabricated nanodevices showed a typicalSchottky behavior.

\section{Summary}

Brief overview on the growth of lead selenide and zinc selenide nanowire growth is presented. Several growth mechanism for the nanowire and kinetics are summarized. Limited measurements on the PbSe nanowire growth supported that models based onimpingements, nucleation size, and alignment of the substrate surface, crystallographic orientation of NW array and thermal and solutal conditions as a whole in both $\mathrm{PbSe}$ and $\mathrm{ZnSe}$ nanowires. Growth of Pb Senanocubes indicated strong orientation in (001) and (111) orientation. Further studies are required to clarify the strong tendency of oriented nanowire growth from building blocks of lead selenidenanocubes or nanoparticles. ZnSe NWs growth by vapor phase deposition indicates high structural and optical quality, further establishing their potential assuitable photodetectors.The metal-semiconductor-metal structures using silver and gold contacts were fabricated for evaluation of photoactivity. The measured results showed an average increase of $10 \mathrm{x}$ in photocurrent and up to $720 \mathrm{x}$ for an individual device. Fabrication still remains the biggest challenge for the low cost production of nanowire based devices.

\section{References}

1. Nguyen P, Ng HT, Meyyappan M (2005) Catalysts metal selection of inorganic Nanowires. Advanced Materials 17(14): 1773-1777.
2. Huang Y, Duan X, Lieber CM (2005) Nanowires for integrated multicolor photonics. Small 1(1):142-147.

3. Hayden O, Agarwal R, Lieber CM (2006) Nanoscale avalanche photodiodes for highly sensitive and spatially resolved photon detection. Nat Mater 5(5): 352-356.

4. Jackson J, Morrison A, Phelan D, Mathewson A (2002) A Novel Silicon Geiger-Mode Avalanche Photodiode. Proceedings IEDM. 32(2): 797 - 800

5. Wu Y, Xiang J, Yang C, Lieber CM (2004) Single crystsal metallic nanowires and semiconductor nanowire heterostructures. Nature 430(6995): 61-65.

6. Lew KK (2004) Structure and electrical properties of trimethyle boron doped Si Nanowires. Applied Physics Letter 85(15): 3101-3103.

7. King M , McLaughlin S, Kahler D, Berghmans A, Wagner B (2010) Nanowire Based Photodetectors: Growth and Development of ChalcogenideNanostructured Detectors. In: Thomas George, M. Saif Islam, Achyut K. Dutta (Eds.), SPIE: Micro- and Nanotechnology Sensors, Systems, and Applications III.

8. Knuteson DJ, Aziz M, Singh NB, Kanner G, Green K et al. (2012) Nanomorphology of ZnSe on patterned substrates. In: Guo R (Ed.), Photonic Fiber and Crystal Devices, SPIE.

9. Cho KS, Talapin DV, Gaschler W, Murray CB (2005) Designing PbSe nanowires and nanorings through oriented attachment of nanoparticles. J Am Chem Soc 127(19): 7140-7147.

10. Parker RL, Anderson RL, Hardy SC (1963) Growth and evaporation 
kinetics and surface diffusion of $\mathrm{K}$ and $\mathrm{Hg}$ whiskers. Applied Physics Letters 3(6): 93 .

11. Givargizov EI, Cherno AA (1973) Rate of whisker growth by the vaporliquid-crystal mechanism and the role of surface energy. Sov Phys Crystallogr 18: 89.

12. Johansson J, Svensson CP, Martensson T, Samuelson L, Seifert W (2005) Mass transport model for semiconductor nanowire growth. J. Phys Chem B 109(28): 13567-13571.

13. Schmidt V, Senz S, Gosele U (2007) Diameter dependence of the growth velocity of silicon nanowires synthesized via the vapor-liquid-solid mechanism. Phys Rev B 75045335.

14. Johansson J, Wacaser BA, Dick KA, Seifert W (2006) Growth related aspects of epitaxial Nanowires. Nanotechnology 17(11): S355.

15. Dubrovskii VG, Sibirev NV, Cirlin GE, Harmand JC, Ustinov VM (2006) Theoretical analysis of the vapor-liquid-solid mechanism of nanowire growth during molecular beam epitaxy. Phys Rev E Stat Nonlin Soft Matter Phys 73(2 Pt 1): 021603.

16. Wagner B, Singh NB, McLaughlin S, Berghmans A, Kahler D et al. (2009) Effect of growth parameters on the morphology and resistivity of PbSe. Journal of Crystal Growth 311(4): 1080-1086.

17. Gudiksen MS, Wang J, Lieber CM (2001) Synthetic control of the diameter and length of single crystal semiconductor Nanowires. J Phys Chem 105(19): 4062-4062. 